\title{
PICARD SETS ADMITTING EXCEPTIONALLY RAMIFIED MEROMORPHIC FUNCTIONS
}

\author{
By YŨ Hashimoto and Kikuji Matsumoto
}

1. For a totally disconnected compact set $E$ in the extended $z$-plane $\hat{\boldsymbol{C}}$, we denote by $M_{E}$ the totality of meromorphic functions, each of which is defined in $\hat{\boldsymbol{C}}-E$ and has $E$ as the set of transcendental singularities. If any function of $M_{E}$ has at most two Picard exceptional values at each singularity $\zeta \in E, E$ is called a Picard set. The existence of perfect Picard sets was shown by means of Cantor sets in Matsumoto [4]. A meromorphic function $f(z)$ of $M_{E}$ is said to be exceptionally ramified at a singularity $\zeta \in E$, if there exist values $w_{k}, 1 \leqq k \leqq q$, and positive integers $\nu_{k}, 1 \leqq k \leqq q$, with

$$
\sum_{k=1}^{q}\left(1-\frac{1}{\nu_{k}}\right)>2
$$

such that, in some neighborhood of $\zeta$, the multiplicity of any $w_{k}$-point of $f(z)$ is not less than $\nu_{k}$. In [3], Kurokawa gave Cantor sets $E$ for which each function of $M_{E}$ cannot be exceptionally ramified at any singularity $\zeta \in E$. Here we remark that such sets $E$ are Picard sets. The purpose of this note is to show that, contrary to the case of isolated singularities, there exist Picard sets $E$ for which there exist meromorphic functions of $M_{E}$ being exceptionally ramified at each singularity $\zeta \in E$.

2. Let $\Delta$ be the 5 -sheeted covering surface of the extended $\omega$-plane with two branch points of order 4 over 1 and -1 . For $n \geqq 0$, we slit each of four sheets other than the first sheet of $\Delta$ along the positive real axis from $\mu_{n}, \mu_{n}>1$, to $\infty$ and denote the resulting surface by $\Delta_{n}$. For $n \geqq 1$, we further slit the first sheet of $\Delta_{n}$ along the positive real axis from $\mu_{n-1}$ to $\infty$ and prepare $4^{n}$ replicas of the resulting surface, which we denote by $\Delta_{n, k}, 1 \leqq k \leqq 4^{n}$.

Now we connect $\Delta_{1, k}, 1 \leqq k \leqq 4$, with $\Delta_{0}$ crosswise across the four slits from $\mu_{0}$ to $\infty$ one by one, and obtain a surface $F_{1}$ with $4^{2}$ slits. Next we connect $\Delta_{2, k}, 1 \leqq k \leqq 4^{2}$, with $F_{1}$ crosswise across the $4^{2}$ slits from $\mu_{1}$ to $\infty$ one by one and obtain a surface $F_{2}$ with $4^{3}$ slits. Continue these constructions inductively. Namely we connect $\Delta_{n+1, k}, 1 \leqq k \leqq 4^{n+1}$, with $F_{n}$ crosswise across the $4^{n+1}$ slits from $\mu_{n}$ to $\infty$ one by one and obtain a surface $F_{n+1}$ with $4^{n+2}$ slits. We denote the limit surface by $F$.

Received October 14, 1988. 
By construction, the surface $F$ is planar, so that there exists a one to one conformal mapping $z=\phi(p)$ of $F$ onto a domain $G$ of the extended $z$-plane $\hat{\boldsymbol{C}}$. Now we set $E=\widehat{\boldsymbol{C}}-G$ and have the following

THEOREM. If $\lim _{n \rightarrow \infty}\left(\mu_{n} / \mu_{n-1}\right)=\infty$, then $E$ is a Picard set for which there exists a meromorphic function of $M_{E}$ being exceptionally ramified at each $\zeta \in E$.

3. We denote by $\pi(p)$ the projection of $F$ to the extended $\omega$-plane. Then the composite function $\pi \circ \phi^{-1}(z)$ is exceptionally ramified in $G$ and belongs to $M_{E}$. Hence it is sufficient for us to prove that $E$ is a Picard set.

Contrary suppose that there exists a meromorphic function $f(z)$ of $M_{E}$ omitting three values 0,1 and $\infty$ in a neighborhood of some singularity $\zeta_{0} \in E$. We consider $\Delta_{n, k}$ defined in 2 as a subdomain of $F$ and denote by $\Gamma_{n, k}$ its boundary corresponding to the slit from $\mu_{n-1}$ to $\infty$ and by $\Gamma_{n+1,4 k-3}, \Gamma_{n+1,4 k-2}$, $\Gamma_{n+1,4 k-1}$ and $\Gamma_{n+1,4 k}$ those corresponding to the four slits from $\mu_{n}$ to $\infty$. Then $\Delta_{n-1, \ell}, k=4 \ell-j(0 \leqq j \leqq 3)$, has $\Gamma_{n, k}$ as a boundary in common with $\Delta_{n, k}$ and we can take a doubly connected domain $A_{n, k}$ in $\Delta_{n-1, \ell} \cup \Gamma_{n, k} \cup \Delta_{n, k}$ with $\pi\left(A_{n, k}\right)$ $\subset\{\omega ;|\omega|>1\}$ which is mapped onto the annulus $1<|\xi|<\mu_{n-1}{ }^{2}$ with $|\xi|=\mu_{n-1}$ as the image of $\Gamma_{n, k}$. For each $n \geqq 1$, the doubly connected domains $\left\{A_{n, k}\right\}$, $1 \leqq k \leqq K(n)=4^{n}$, separate $\left\{A_{n-1, k}\right\}, 1 \leqq k \leqq K(n-1)$, from the ideal boundary of $F$ and $\sum_{m=1}^{n} \log \mu_{m-1}{ }^{2}-(1 / 2) \log K(n)=2 \sum_{m=1}^{n} \log \mu_{m-1}-n \log 2 \rightarrow \infty(n \rightarrow \infty)$ from our assumption $\lim _{n \rightarrow \infty}\left(\mu_{n} / \mu_{n-1}\right)=\infty$, and so we see from the Pfluger-Mori criterion that $F$ is an open Riemann surface of $O_{A B}$, that is, $E$ is an $N_{\mathfrak{B}}$-set (see [5]). Therefore any function of $M_{E}$ cannot be bounded at any singularity $\zeta \in E$. Now we consider the function $\tilde{f}(p)=f(\phi(p))$ on $F$.

Lemma 1 (Carleson [2] and Matsumoto [4]). Let $w=g(\xi)$ be a single-valued regular function in an annulus $1<|\xi|<\mu^{2}$ omitting two values 0 and 1 . Then, the diameter of the image of $|\xi|=\mu$ by $g(\xi)$ with respect to the chordal distance is dominated by $A / \mu$ for sufficiently large $\mu$. Here, $A$ is a positive constant not depending on $\mu$ and $g(\xi)$.

We take $\Gamma_{n_{0}, k_{0}}$ such that $\gamma_{n_{0}, k_{0}}=\phi\left(\Gamma_{n_{0}, k_{0}}\right)$ surrounds $\zeta^{0}$, where we may assume that $f(z)$ omits 0,1 and $\infty$ in the part of $G$ surrounded with $\gamma_{n_{0}, k_{0}}$, that is, $\tilde{f}(p)$ omits, 0,1 and $\infty$ in the end $F_{0}$ of $F$ which has $\Gamma_{n_{0}, k_{0}}$ as its relative boundary. From now on, we deal only with $\Delta_{n, k}$ contained in the end $F_{0}$ and see from this lemma that the diameter of the image $\tilde{f}\left(\Gamma_{n, k}\right)$ with respect to the chordal distance is dominated by $A / \mu_{n-1}$ for sufficiently large $n$. Hence we can take a spherical disc $D_{n, k}$ of chordal radius $\delta_{n}=A / \mu_{n-1}$ containing the image $\tilde{f}\left(\Gamma_{n, k}\right)$.

4. We may assume that $\delta_{n}<1 / 20$ for $n \geqq n_{0}$. Then, since any component 
of $D=D_{n, k} \cup D_{n+1,4 k-3} \cup D_{n+1,4 k-2} \cup D_{n+1,4 k-1} \cup D_{n+1,4 k}$ can contain at most one of 0,1 and $\infty$ and $\tilde{f}(p)$ omits them in $\Delta=\Delta_{n, k}$, we have the following cases:

(A) The set $D$ is connected. We cover $D$ with a disc $\hat{D}$ of radius at most $5 \delta_{n}$ and have $\tilde{f}(\Delta) \subset \hat{D}$. In this case, we say that $\Delta_{n, k}$ is degenerate $(\tilde{f})$.

(B) The set $D$ is not connected and is covered with the following discs, which are disjoint by pair and each of which covers at least one component of $D$. Here we may assume that there are no branch points of the Riemannian image of $\Delta$ over the boundary circles of these discs. The function $\tilde{f}(p)$ takes each value outside their union at least once and the same times, say $s \geqq 1$ times. In this case, we say that $\Delta_{n, k}$ is nondegenerate $(\tilde{f})$.

(B1) Discs $\hat{D}_{0}, \hat{D}_{1}$ and $\hat{D}_{\infty}$ of radius at most $6 \delta_{n}$ and with centers at 0,1 and $\infty$ respectively.

(B2) Discs $\hat{D}_{0}, \hat{D}_{1}$ and $\hat{D}_{\infty}$ and one more disc $\hat{D}_{a}$ of radius at most $2 \delta_{n}$ and with center at $a$.

(B3) Discs $\hat{D}_{0}, \hat{D}_{1}$ and $\hat{D}_{\infty}$ and two more discs $\hat{D}_{a}$ and $\hat{D}_{b}$ of radius $\delta_{n}$ and with centers at $a$ and $b$ respectively.

We shall go into details about Riemannian image of $\Delta$ under $\tilde{f}(p)$. First we shall prove the following

LEMMA 2. The harmonic moduli of doubly connected domains in $\Delta=\Delta_{n, k}$ which separate two of five boundary curves of $\Delta$ from the remaining three have an upper bound $K$ not depending on $n$ and $k, 1 \leqq k \leqq 4^{n}$.

Proof. Map $\Delta$ by $u=\{(\omega+1) /(\omega-1)\}^{1 / 5}$. The image of $\Delta$ is the extended $u$-plane with a slit from 1 to $\left\{\left(\mu_{n-1}+1\right) /\left(\mu_{n-1}-1\right)\right\}^{1 / 5}$ and four slits from $e^{2 n \pi \imath / 5}$ to $e^{2 m \pi 2 / 5}\left\{\left(\mu_{n}+1\right) /\left(\mu_{n}-1\right)\right\}^{1 / 5}, 1 \leqq m \leqq 4$. The image of any doubly connected domain of our lemma separates two of these five slits from the remaining three, consequently, two of the five points $\left\{e^{2 m \pi \imath / 5}\right\}_{m=0}^{4}$ from the remaining three. Therefore it is sufficient for us to find an upper bound out for doubly connected domains which separate 1 and $e^{2 \pi \imath / 5}$ from $e^{4 \pi \imath / 5}, e^{6 \pi \imath / 5}$ and $e^{8 \pi i / 5}$ or $1, e^{2 \pi \imath / 5}$ and $e^{6 \pi \imath / 5}$ from $e^{4 \pi \imath / 5}$ and $e^{8 \pi \imath / 5}$. It is well-known that the doubly connected domain $\widehat{\boldsymbol{C}}-\left\{e^{i \theta} ; 0 \leqq \theta \leqq 2 \pi / 5\right\} \cup\left\{e^{i \theta} ; 4 \pi / 5 \leqq \theta \leqq 8 \pi / 5\right\}$ gives the maximum harmonic modulus among the doubly connected domains separating 1 and $e^{2 \pi \imath / 5}$ from $e^{4 \pi \imath / 5}$ and $e^{8 \pi 2 / 5}$. Thus we can take the harmonic modulus of this domain as $K$.

(B1) We set $\Omega=\hat{\boldsymbol{C}}-\hat{D}_{0} \cup \hat{D}_{1} \cup \hat{D}_{\infty}$. The inverse image $\tilde{f}^{-1}(\Omega)$ consists of at most two components, because the connectivity of any component cannot be less than 3. Suppose that $\tilde{f}^{-1}(\Omega)$ consists of two subdomains $\hat{\Delta}_{1}$ and $\hat{\Delta}_{2}$ of $\Delta$. Then $\hat{\Delta}_{1}$ and $\hat{\Delta}_{2}$ are triply connected. Let $S_{1}$ and $S_{2}$ be the Riemannian image of $\hat{\Delta}_{1}$ and $\hat{\Delta}_{2}$ and $s_{1}$ and $s_{2}$ be the numbers of sheets of $S_{1}$ and $S_{2}$. Expressing by $\rho$ the Euler characteristics, we have

$$
\rho(\Omega)\left(s_{1}+s_{2}\right)-\left(v_{1}+v_{2}\right)=\rho\left(\hat{\Delta}_{1}\right)+\rho\left(\hat{\Delta}_{2}\right)=-2,
$$

where $v_{1}$ and $v_{2}$ denote the ramification indices of $S_{1}$ and $S_{2}$. Since $\rho(\Omega)=-1$, $s_{1}+s_{2}+v_{1}+v_{2}=2$, so that $s_{1}=s_{2}=1$ and $v_{1}=v_{2}=0$. A component of the open set 
$\Delta-\overline{\hat{\Delta}_{1} \cup \hat{\Delta}_{2}}$ is triply connected and its image is contained in some of $\hat{D}_{0}, \hat{D}_{1}$ and $\hat{D}_{\infty}$, say $\hat{D}_{0}$. The inverse image of any doubly connected domain in $S_{1}$ separating $\hat{D}_{0}$ from $\hat{D}_{1}$ and $\hat{D}_{\infty}$ separates two of five boundary curves of $\Delta$ from the remaining three. By Lemma 2, this is impossible for sufficiently large $n$. Thus we see that $\tilde{f}^{-1}(\Omega)$ consists of a subdomain $\hat{\Delta}$ of $\Delta$, where the connectivity of $\hat{\Delta}$ is 3,4 or 5 . Let $S$ be the Riemannian image of $\hat{\Delta}$ and $v$ be the ramification index of $S$. Then $\rho(\Omega) s-v=\rho(\hat{\Delta})$, that is, $s+v=-\rho(\hat{\Delta})$.

If $\hat{\Delta}$ is triply connected, we have $s+v=1$, so that $s=1$ and $v=0$. There is a boundary curve $\hat{\Gamma}$ of $\hat{\Delta}$ which surrounds at least two of five boundary curves of $\Delta$. Suppose that $\tilde{f}(\hat{\Gamma})=\partial \hat{D}_{0}$. Then the inverse image of any doubly connected domain in $S$ separating $\hat{D}_{0}$ from $\hat{D}_{1}$ and $\hat{D}_{\infty}$ separates two of five boundary curves of $\Delta$ from the remaining three. By Lemma 2, this is impossible for sufficiently large $n$. We can show similarly that the case where $\hat{\Delta}$ is quadruply connected does not occur.

In the case that $\hat{\Delta}$ is quintuply connected, we have $s+v=3$, so that $s=3$ and $v=0$ or $s=2$ and $v=1$. We denote by $\sigma_{0}, \sigma_{1}$ and $\sigma_{\infty}$ the numbers of boundary curves of $\hat{\Delta}$ mapped onto $\partial \hat{D}_{0}, \partial \hat{D}_{1}$ and $\partial \hat{D}_{\infty}$ respectively, where we may assume $\sigma_{0} \geqq \sigma_{1} \geqq \sigma_{\infty}=1$. Then we have following three cases.

$\begin{array}{ccccc}\sigma_{0} & \sigma_{1} & \sigma_{\infty} & s & v \\ 3 & 1 & 1 & 3 & 0 \\ \{1,1,1\} & \{3\} & \{3\} & & \\ 2 & 2 & 1 & & \\ \{1,1\} & \{1,1\} & \{2\} & 2 & 1 \\ 2 & 2 & 1 & 3 & 0 \\ \{1,2\} & \{1,2\} & \{3\} & 3 & \end{array}$

Here we write under $\sigma_{0}, \sigma_{1}$ and $\sigma_{\infty}$ the winding numbers of the images of boundary curves of $\hat{\Delta}$ with respect to the centers of $\hat{D}_{0}, \hat{D}_{1}$ and $\hat{D}_{\infty}$ respectively. For instance $\underset{\{1,2\}}{\sigma_{1}}$ shows that there are two boundary curves of $\hat{\Delta}$ mapped onto $\partial \hat{D}_{1}$ and one of them has the image winding once around 1 , while the other has the image winding twice.

(B2) The inverse image $\tilde{f}^{-1}(\Omega)$ consists of a subdomain of $\Delta$. We take all relatively noncompact components of $\tilde{f}^{-1}\left(\hat{D}_{a}\right)$ with respect to $\Delta$ off from $\tilde{f}^{-1}(\Omega)$ and have a subdomain $\hat{\Delta}$ of $\Delta$, whose connectivity is 4 or 5 . Let $S$ denote the Riemannian image of $\hat{\Delta}$ under $\tilde{f}(p)$ and $v$ be the ramification index of $S$. Denoting by $s_{a}$ the number of sheets of $S$ over $\hat{D}_{a}$ and by $v_{a}$ the ramification index of the part of $S$ over $\hat{D}_{a}$, we have

$$
\left\{\rho\left(\Omega-\overline{\hat{D}}_{a}\right) s-\left(v-v_{a}\right)\right\}+\left\{\rho\left(\hat{D}_{a}\right) s_{a}-v_{a}\right\}=\rho(\hat{\Delta}),
$$

that is, $2 s-s_{a}+v=-\rho(\hat{\Delta})$. In the case that $\hat{\Delta}$ is quadruply connected, we have $s=1, \quad s_{a}=0$ and $v=0$, but we see similarly as in (B1) that this case does not occur for sufficiently large $n$. Let $\hat{\Delta}$ be quintuply connected. Then $2 s-s_{a}+v$ 
$=3$ and we have $s=2, s_{a}=1$ and $v=0$. Denoting by $\sigma_{a}$ the number of boundary curves of $\hat{\Delta}$ mapped onto $\partial \hat{D}_{a}$, we have the following case.

$\begin{array}{cccccc}\sigma_{0} & \sigma_{0} & \sigma_{\infty} & \sigma_{a} & s & v \\ 2 & 1 & 1 & 1 & 2 & 0 \\ \{1,1\} & \{2\} & \{2\} & \{1\} & & \end{array}$

(B3) We take all relatively noncompact components of $\tilde{f}^{-1}\left(\hat{D}_{a}\right)$ and $\tilde{f}^{-1}\left(\hat{D}_{b}\right)$ with respect to $\Delta$ off from $\tilde{f}^{-1}(\Omega)$ and have a subdomain $\hat{\Delta}$ of $\Delta$. The domain $\hat{\Delta}$ is quintuply connected. Hence we have

$$
\left\{\rho\left(\Omega-\overline{\hat{D}_{a} \cup \hat{D}_{b}}\right) s-\left(v-v_{a}-v_{b}\right)\right\}+\left\{\rho\left(\hat{D}_{a}\right) s_{a}-v_{a}\right\}+\left\{\rho\left(\hat{D}_{b}\right) s_{b}-v_{b}\right\}=-3,
$$

that is, $3 s-s_{a}-s_{b}+v=3$, so that $s=1, s_{a}=s_{b}=0$ and $v=0$.

$\begin{array}{ccccccc}\sigma_{0} & \sigma_{1} & \sigma_{\infty} & \sigma_{a} & \sigma_{b} & s & v \\ 1 & 1 & 1 & 1 & 1 & 1 & 0 \\ \{1\} & \{1\} & \{1\} & \{1\} & \{1\} & & \end{array}$

5. Suppose that all $\Delta_{n, k}$ contained in the end $F_{0}$ are degenerate $(\tilde{f})$. Then for $\Delta_{n, k}$ in $F_{0}, \tilde{f}\left(\Delta_{n, k}\right)$ is covered with a disc $\hat{D}_{n, k}$ of radius $5 \delta_{n}$. For each $\Delta_{n_{0}+1, k}$ in $F_{0}, \hat{D}_{n_{0}, k_{0}} \cup \hat{D}_{n_{0}+1, k} \neq \phi$, so that $\tilde{f}\left(\Delta_{n_{0}, k_{0}} \cup\left(\cup_{k}^{\prime} \Delta_{n_{0}+1, k}\right)\right)$ is covered with the disk $\widetilde{D}_{1}$ of radius $5 \delta_{n_{0}}+10 \delta_{n_{0+1}}<(1 / 4)\{1+(1 / 2)\}$ and with the same center $a_{0}$ as $\hat{D}_{n_{0}, k_{0}}$, where $\bigcup_{k}^{\prime} \Delta_{n_{0}+1, k}$ means the union taken over all the $\Delta_{n_{0}+1, k}$ 's in $F_{0}$ and we assume $\delta_{n_{0}}<1 / 20$ and $\delta_{n+1} / \delta_{n}<1 / 4$ for $n \geqq n_{0}$. Suppose now that $\tilde{f}\left(\Delta_{n_{0}, k_{0}} \cup\left(\bigcup_{p=1}^{m}\left(\bigcup_{k}^{\prime} \Delta_{n_{0}+p, k}\right)\right)\right)$ is covered with the disc $\tilde{D}_{m}$ of radius $5 \delta_{n_{0}}+10 \sum_{p=1}^{m} \delta_{n_{0}+p}$ $<(1 / 4) \sum_{p=0}^{m}\left(1 / 2^{p}\right)$ and with center at $a_{0}$. Then $\tilde{f}\left(\Delta_{n_{0}, k_{0}} \cup\left(\bigcup_{p=1}^{m+1}\left(\bigcup_{k}^{\prime} \Delta_{n_{0}+p, k}\right)\right)\right)$ is covered with the disc $\tilde{D}_{m+1}$ of radius $5 \delta_{n_{0}}+10 \sum_{p=1}^{m+1} \delta_{n_{0}+p}<(1 / 4) \sum_{p=0}^{m+1}\left(1 / 2^{p}\right)$ and with center at $a_{0}$, because $\widetilde{D}_{m} \cap \hat{D}_{n_{0}+m+1, k} \neq \phi$ for each $\Delta_{n_{0}+m+1, k}$ in $F_{0}$. Thus we conclude that $\tilde{f}\left(F_{0}\right)$ is covered with the disc of radius $(1 / 4) \sum_{p=0}^{\infty}\left(1 / 2^{p}\right)=1 / 2$ and with center at $a_{0}$. This means that $f(z)$ is bounded in the part of $G$ surrounded with $\gamma_{n_{0}, k_{0}}$ and is impossible as mentioned in 3 . Now we see that there are infinitely many $\Delta_{n, k}$ in $F_{0}$ being nondegenerate $(\tilde{f})$.

6. We take such a domain $\Delta_{n, k}$ and consider the subdomain $\hat{\Delta}_{n, k}$ of $\Delta_{n, k}$. As we saw in $4, \hat{\Delta}_{n, k}$ is quintuply connected and its five boundaries $\check{\Gamma}_{n, k}$ and $\left\{\hat{\Gamma}_{n+1,4 k-j}\right\}_{j=0}^{3}$ are homotopic to those $\Gamma_{n, k}$ and $\left\{\Gamma_{n+1,4 k-j}\right\}_{j=0}^{3}$ of $\Delta_{n, k}$ respectively. Further, at least two of them have the image curves winding once around 0 , 1 or $\infty$, so that one of $\left\{\hat{\Gamma}_{n+1,4 k-j}\right\}_{j=0}^{3}$, say $\hat{\Gamma}_{n+1,4 k}$ has the image curve winding once around 0,1 or $\infty$, say 0 . The adjacent $\Delta_{n+1,4}$ is degenerate $(\tilde{f})$. In fact, suppose that it is nondegenerate $(\tilde{f})$. Then the boundary curve $\hat{\Gamma}_{n+1,4 k}$ of $\hat{\Delta}_{n, k}$ 
and that $\check{\Gamma}_{n+1,4 k}$ of $\hat{\Delta}_{n+1,4 k}$ are both homotopic to $\Gamma_{n+1,4 k}$ and bound a subdomain of $\Delta_{n, k} \cup \Gamma_{n+1,4} \cup \Delta_{n+1,4 k}$, so that $\tilde{f}(p)$ takes the value 0 there contradicting our assumption that $\tilde{f}(p)$ omits 0,1 and $\infty$. Here we give two lemmas.

LemMA 3. If $\Delta_{n, k}$ is degenerate $(\tilde{f})$, there are four curves $\left\{\tilde{\Gamma}_{n+1,4 k-j}\right\}_{j=0}^{3}$ in $\Delta_{n, k}$ such that, for each $j, 0 \leqq j \leqq 3, \tilde{\Gamma}_{n+1,4 k-\jmath}$ is homotopic to $\Gamma_{n+1,4 k-j}$ and the diameter of its image $\tilde{f}\left(\tilde{\Gamma}_{n+1,4 k-j}\right)$ with respect to the chordal distance is dominated by $\tilde{A} \delta_{n} \delta_{n+1}=o\left(\delta_{n}{ }^{2}\right)$, where $\tilde{A}$ is a positive constant.

Proof. A disc $\hat{D}_{n, k}$ of radius at most $5 \delta_{n}$ covers the image $\tilde{f}\left(\Delta_{n, k}\right)$. We map $\Delta_{n, k}$ by the mapping $u=\{(\omega+1) /(\omega-1)\}^{1 / 5}$. The image of $\Delta_{n, k}$ is the extended $u$-plane with a radial slit $R_{n, k}$ from 1 to $\left.\left\{\left(\mu_{n-1}+1\right) / \mu_{n-1}-1\right)\right\}^{1 / 5}$, the image of $\Gamma_{n, k}$, and four slits $\left\{R_{n+1,4 k-j}\right\}_{j=0}^{3}$ from $e^{2(j+1) \pi \imath / 5}$ to $e^{2(j+1) \pi \imath / 5}\left\{\left(\mu_{n}+1\right) /\right.$ $\left.\left(\mu_{n}-1\right)\right\}^{1 / 5}, 0 \leqq j \leqq 3$, the images of $\left\{\Gamma_{n+1,4 k-j}\right\}_{j=0}^{3}$. We take circles $\left\{C_{n+1,4 k-j}\right\}_{j=0}^{3}$ of radius $\mu_{n-1} / \mu_{n}=\delta_{n+1} / \delta_{n}$ and with centers at $e^{2(\jmath+1) \pi \imath / 5}, 0 \leqq j \leqq 3$. We show that the inverse images $\left\{\tilde{\Gamma}_{n+1,4 k-j}\right\}_{j=0}^{3}$ of these circles $\left\{C_{n+1,4 k-j}\right\}_{j=0}^{3}$ satisfy the conditions of our lemma. We set $h(u)=\tilde{f}\left(\left(u^{5}+1\right) /\left(u^{5}-1\right)\right)$ and apply the Cauchy integral formula to $h(u)$ in the domain $\{u ;|u|<R\}-R_{n, k} \cup\left(\bigcup_{\jmath=0}^{3} R_{n+1,4 k-\jmath}\right)$, where we take $R>0$ sufficiently large. Then we have

$$
\frac{d h}{d u}\left(u_{0}\right)=\frac{1}{2 \pi \imath}\left\{\int_{R_{n, k}}+\sum_{\jmath=0}^{3} \int_{R_{n+1,4 k-\jmath}}+\int_{|u|=R}\right\} \frac{h(u)}{\left(u-u_{0}\right)^{2}} d u .
$$

We take $u_{0} \in C_{n+1,4 k-\jmath}$. Here we may assume that the center of the disc $D_{n+1,4 k-\jmath}$ is 0 . The disc $D_{n+1,4 k-\jmath}$ was given in 4 , and, as we showed there, $D_{n+1,4 k-\jmath} \supset \tilde{f}\left(\Gamma_{n+1,4 k-j}\right), D_{n+1,4 k-j} \subset \hat{D}_{n, k}$, and its radius is equal to $\delta_{n+1}$. We remark that $\hat{D}_{n, k}$ is covered with the disc of radius $10 \delta_{n}$ and with center at 0 . Since $\tilde{f}\left(\Delta_{n, k}\right) \subset \hat{D}_{n, k},|h(u)| \leqq 20 \delta_{n}$ and

$$
\left|\int_{|u|=R} \frac{h(u)}{\left(u-u_{0}\right)^{2}} d u\right| \leqq 20 \delta_{n} \frac{2 \pi R}{\left(R-\left|u_{0}\right|\right)^{2}} \longrightarrow 0 \quad(R \rightarrow \infty) .
$$

Since $\left(\delta_{n+1} / \delta_{n}\right) \rightarrow 0(n \rightarrow \infty)$, we may assume that the distance between $C_{n+1,4 k-\jmath}$ and $R_{n, k}$ or $R_{n+1,4 k-\ell}, 0 \leqq \ell \leqq 3$ and $\neq \ell j$, are not less than $\sin (\pi / 5)$. We have

$$
\left|\int_{R_{n, k} k} \frac{h(u)}{\left(u-u_{0}\right)^{2}} d u\right| \leqq 20 \delta_{n} \frac{2\left|R_{n, k}\right|}{\sin ^{2}(\pi / 5)}
$$

and

$$
\left|\int_{R_{n+1,4 k-\ell}} \frac{h(u)}{\left(u-u_{0}\right)^{2}} d u\right| \leqq 20 \delta_{n} \frac{2\left|R_{n+1,4 k-\ell}\right|}{\sin ^{2}(\pi / 5)}
$$

where $\left|R_{n, k}\right|$ and $\left|R_{n+1,4 k-\ell}\right|$ denotes the lengths of the radial slits $R_{n, k}$ and

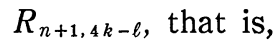

$$
\left|R_{n, k}\right|=\left(\frac{\mu_{n-1}+1}{\mu_{n-1}-1}\right)^{1 / 5}-1 \leqq \frac{3}{5 \mu_{n-1}} \leqq \frac{3}{5 A} \delta_{n}
$$


and

$$
\left|R_{n+1,4 k-\ell}\right|=\left(\frac{\mu_{n}+1}{\mu_{n}-1}\right)^{1 / 5}-1 \leqq \frac{3}{5 A} \delta_{n+1},
$$

where $A$ is the positive constant in Lemma 1 . We remark that $\tilde{f}\left(\Gamma_{n+1,4 k-j}\right) \subset$ $D_{n+1,4 k-\jmath}$ and hence $|h(u)| \leqq 10 \delta_{n+1}$ for $u \in R_{n+1,4 k-\jmath}$. Since $\left(\delta_{n+1} / \delta_{n}\right)-\left|R_{n+1,4 k-j}\right|$ $\geqq \delta_{n+1} /\left(2 \delta_{n}\right)$ for sufficiently large $n$,

$$
\left|\int_{R_{n+1,4 k-\jmath}} \frac{h(u)}{\left(u-u_{0}\right)^{2}} d u\right| \leqq 10 \delta_{n+1} \frac{2\left|R_{n+1,4 k-j}\right|}{\left\{\left(\delta_{n+1} / \delta_{n}\right)-\left|R_{n+1,4 k-j}\right|\right\}^{2}} \leqq \frac{48}{A} \delta_{n}{ }^{2} .
$$

Thus we have

$$
\left|\frac{d h}{d u}\left(u_{0}\right)\right| \leqq \frac{1}{2 \pi}\left(\frac{24}{A \sin ^{2}(\pi / 5)} \delta_{n}{ }^{2}+3 \frac{24}{A \sin ^{2}(\pi / 5)} \delta_{n} \delta_{n+1}+\frac{48}{A} \delta_{n}{ }^{2}\right) \leqq \frac{\tilde{A}}{2 \pi} \delta_{n}{ }^{2},
$$

so that the diameter of the image $h\left(C_{n+1,4 k-j}\right)=\tilde{f}\left(\tilde{\Gamma}_{n+1,4 k-j}\right)$ is dominated by

$$
\int_{C_{n+1,4 k-\jmath}}\left|\frac{d h}{d u}(u)\right||d u| \leqq \frac{\tilde{A}}{2 \pi} \delta_{n}{ }^{2} \cdot 2 \pi \frac{\delta_{n+1}}{\delta_{n}}=\tilde{A} \delta_{n} \delta_{n+1} .
$$

LEMMA 4. The harmonic modulus of any doubly connected domain in $\Delta=$ $\Delta_{n, k} \cup \Gamma_{n+1,4 k} \cup \Delta_{n+1,4 k}$ which separates $\Gamma_{n, k}$ and $\left\{\Gamma_{n+1,4 k-j}\right\}_{j=1}^{3}$ from $\left\{\Gamma_{n+2,16 k-j}\right\}_{j=0}^{3}$ is dominated by $-2 \log M \delta_{n+1}$, where $M$ is a positive constant.

Proof. We map $\Delta$ by the mapping $u=\{(\omega+1) /(\omega-1)\}^{1 / 5}$, where we take a branch of the mapping function suitably so that the image $R_{n+1,4 k}$ of $\Gamma_{n+1,4 k}$ comes on the positive real axis, and have a two-sheeted covering surface over the extended $u$-plane with branch points over 1 and $r=\left\{\left(\mu_{n}+1\right) /\left(\mu_{n}-1\right)\right\}^{1 / 5}$. Further we map this surface by $v=(u-1) /(u-r)$ and have alsotwo-sheeted covering surface over the extended $v$-plane branching over 0 and $\infty$. We consider that the covering surface is given by connecting two extended $v$-plane crosswise across the negative real axis. The boundary curves $\Gamma_{n, k}$ and $\left\{\Gamma_{n+1.4 k-j}\right\}_{j=1}^{3}$ are mapped into the first sheet and the boundary curves $\left\{\Gamma_{n+2,16 k-j}\right\}_{j_{00}^{3}}^{3}$ are mapped into the second sheet. The image of a doubly connected domain in $\Delta$ of our lemma is a doubly connected domain in the surface separating these image curves of the first sheet from those of the second sheet. The image curves in the first sheet and the second sheet both contain two points with projections $v_{0}=\left(e^{2 \pi 2 / 5}-1\right) /\left(e^{2 \pi 2 / 5}-r\right)$ and $\bar{v}_{0}$ and hence our image domain separates these two points of the first sheet from those of the second sheet. It is known that the two-sheeted covering surface deleted the circular arcs of the first and the second sheets over $\left\{v=\left|v_{0}\right| e^{i \theta} ;-\arg v_{0} \leqq \theta \leqq \arg v_{0}\right\}$ gives the maximum harmonic modulus among such doubly connected domains. Its harmonic modulus is equal to two times the harmonic modulus of the Mori extremal domain, the extended plane deleted the negative real axis and the circular $\operatorname{arc}\left\{e^{i \theta} ;-\arg v_{0} \leqq \theta \leqq\right.$ $\left.\arg v_{0}\right\}$. Setting $\lambda=2 \sin \left(\arg v_{0}\right)$, we see that this harmonic modulus is dominated by $\log (16 / \lambda)$ (see Ahlfors [1]). Since $\lambda \geqq M^{\prime} / \mu_{n}=\left(M^{\prime} / A\right) \delta_{n+1}$ for some 
positive constant $M^{\prime}$, we see that $-2 \log M \delta_{n+1}, \quad M=M^{\prime} /(16 A)$, gives an upper bound for harmonic moduli of our doubly connected domains.

The domain $\Delta_{n+1,4 k}$ is degenerate $(\tilde{f})$ and hence by Lemma 3 , there are four curves $\left\{\tilde{\Gamma}_{n+2,16 k-j}\right\}_{j=0}^{3}$ such that, for each $j, 0 \leqq j \leqq 3, \tilde{\Gamma}_{n+2,16 k-j}$ is homotopic to $\Gamma_{n+2,16 k-\jmath}$ and the image $\tilde{f}\left(\tilde{\Gamma}_{n+2,16 k-j}\right)$ is covered with a disc $\tilde{D}_{n+2,16 k-\jmath}$ of radius $\tilde{A} \delta_{n+1} \delta_{n+2}$. We denote by $\tilde{\Delta}_{n+1,4 k}$ the subdomain of $\Delta_{n, k} \cup \Gamma_{n+1,4 k} \cup$ $\Delta_{n+1,4 k}$ bounded by $\hat{\Gamma}_{n+1,4 k}$ and $\left\{\tilde{\Gamma}_{n+2,16 k-j}\right\}_{j=0}^{3}$ and by $S$ and $\tilde{S}$ the Riemanian image of $\hat{\Delta}_{n, k}$ and $\hat{\Delta}_{n, k} \cup \hat{\Gamma}_{n+1,4} \cup \tilde{\Delta}_{n+1,4}$ respectively.

We denote by $\left[w_{1}, w_{2}\right]$ the chordal distance between $w_{1}$ and $w_{2}$. If the case (2) of (B1) occurs for $\Delta_{n, k}, S$ has a branch point over $c \notin \hat{D}_{0} \cup \hat{D}_{1} \cup \hat{D}_{\infty}$. We may assume that $[c, 0] \geqq \sqrt{2} / 4$, because, if $[c, 0]<\sqrt{2} / 4$, then $[c, 1] \geqq \sqrt{2} / 4$ and we can proceed with our proof taking a boundary curve $\hat{\Gamma}_{n+1, q}$ in $\left\{\hat{\Gamma}_{n+1,4 k-j}\right\}_{j=0}^{3}$ instead of $\hat{\Gamma}_{n+1,4 k}$ such that its image curve winds once around 1 . If the case (4) of (B2) occurs for $\Delta_{n, k}$ and $[a, 0]<\sqrt{2} / 2-6 \delta_{n}$, then $S$ has a doubly connected domain over $[a, 0]<[w, 0]<\sqrt{2} / 2-6 \delta_{n}$ whose inverse image separates separates two of five boundary curves of $\hat{\Delta}_{n, k}$ from the remaining three. Hence, by Lemma 2 , we have $[a, 0] \geqq e^{-K} / 2 \sqrt{1+\left(e^{-K} / 2\right)^{2}} \geqq \tilde{K}$, where we set $\tilde{K}=$ $\min \left\{e^{-K} / 2 \sqrt{1+\left(e^{-K} / 2\right)^{2}}, \sqrt{ } 2 / 4\right\}$. If the case (5) of (B3) occurs for $\Delta_{n, k}$, then $[a, 0] \geqq \widetilde{K}$ or $[b, 0] \geqq \widetilde{K}$. We assume that $[a, 0] \geqq \widetilde{K}>[b, 0]$. Then $S$ has a doubly connected domain over $[b, 0]+2 \delta_{n}<[w, 0]<\widetilde{K}$ whose inverse image separates two of the boundary curves of $\hat{\Delta}_{n, k}$ from the remaining three. Hence $[b, 0] \geqq \widetilde{K}^{2}$.

Suppose that $\bigcup_{j=0}^{3} \tilde{D}_{n+2,16 k-j} \subset\{w ;[w, 0]<d\}$ for some positive number $d<\tilde{K}^{2}$.

We consider the part of $\tilde{S}$ over the annulus $\left\{w ; d<[w, 0]<\tilde{K}^{2}\right\}$. Then, as we just saw above, its component containing $\tilde{f}\left(\hat{\Gamma}_{n+1,4 k}\right)$ covers the annulus univalently, so that the inverse image of this component is a doubly connected domain in $\Delta_{n, k} \cup \Gamma_{n+1,4 k} \cup \Delta_{n+1,4 k}$ which separates $\Gamma_{n, k}$ and $\left\{\Gamma_{n+1,4 k-j}\right\}_{j=1}^{3}$ from $\left\{\Gamma_{n+2,16 k-j}\right\}_{j=0}^{3}$ and has the harmonic modulus $\log \left(\widetilde{K}^{2} \sqrt{1-d^{2}}\right) /\left(d \sqrt{1-\widetilde{K}^{4}}\right)$. By Lemma 4 , we have $\log \left(\widetilde{K}^{2} \sqrt{1-d^{2}}\right) /\left(d \sqrt{1-\widetilde{K}^{4}}\right) \leqq-2 \log M \delta_{n+1}$. Thus $d \geqq L \delta_{n+1}{ }^{2}$, $L=\left(\widetilde{K}^{2} M^{2}\right) /\left(2 \sqrt{1-\widetilde{K}^{4}}\right)$, and at least one of $\left\{\widetilde{D}_{n+2,16 k-j}\right\}_{j=0}^{3}$, say $\widetilde{D}_{n+2,16 k}$ intersects the circle $[w, 0]=L \delta_{n+1}{ }^{2}=m$. We assume that $\delta_{n+1} / \delta_{n}<\min \{1 /(4 \tilde{A}), 1 / 4\}$ for $n \geqq n_{0}$. Then since $\tilde{A} \delta_{n+1} \delta_{n+2}<\delta_{n+1}{ }^{2} / 4, \widetilde{D}_{n+2,16 k} \subset\{w ;[w, 0] \geqq m / 2\}$. Suppose that the adjacent domain $\Delta_{n+2,16 k}$ is nondegenerate $(\tilde{f})$. Since the boundary curve $\check{\Gamma}_{n+2,16 k}$ of $\hat{\Delta}_{n+2,16 k}$ is homotopic to $\Gamma_{n+2,16 k}$ and hence to $\tilde{\Gamma}_{n+2,16 k}, \check{\Gamma}_{n+2,16 k}$ and $\tilde{\Gamma}_{n+2,16 k}$ bound a doubly connected subdomain of $\Delta_{n+1,4 k} \cup \Gamma_{n+2,16 k} \cup \Delta_{n+2,16 k}$ and their images are contained in the union of a circle $\tilde{f}\left(\check{\Gamma}_{n+2,16 k}\right)$ with center at 0 and $\widetilde{D}_{n+2,16 k}$, so that $\tilde{f}(p)$ takes each value of the inside of the circle deleted $\widetilde{D}_{n+2,16 k}$. This contradicts our assumption that $\tilde{f}(p)$ omits 0,1 and $\infty$. Thus $\Delta_{n+2,16 k}$ is degenerate $(\tilde{f})$. The curves $\tilde{\Gamma}_{n+3,64 k-\jmath}, 0 \leqq j \leqq 3$, of Lemma 3 are covered with discs $\tilde{D}_{n+3,64 k-\jmath}, 0 \leqq j \leqq 3$, of radius $\tilde{A} \delta_{n+2} \delta_{n+3}<m / 64$. Since 
the set $\tilde{D}_{n+2,16 k} \cup\left(\bigcup_{j=0}^{3} \tilde{D}_{n+3,64 k-j}\right)$ is connected and $\tilde{D}_{n+2,16 k} \subset\{w ;[w, 0] \geqq m / 2\}$, we have $\bigcup_{j=0}^{3} \widetilde{D}_{n+3,64 k-j} \subset\left\{w ;[w, 0] \geqq m / 2^{2}\right\}$.

Now we consider the end $F_{0}^{\prime}$ of $F$ with $\Gamma_{n+2,16 k}$ as its relative boundary and suppose that, for some $p \geqq 0,(*)$ all $\Delta_{n+2+p, q}$ 's in $F_{0}^{\prime}$ are degenerate $(\tilde{f})$ and $\bigcup_{q}^{\prime}\left(\bigcup_{j=0}^{3} \widetilde{D}_{n+3+p, 4 q-j}\right) \subset\left\{w ;[w, 0] \geqq m / 2^{p+2}\right\}$, where $\bigcup_{q}^{\prime}$ means the union taken over all the $\Delta_{n+2+p, q}$ 's in $F_{0}{ }^{\prime}$. We repeat the above argument taking $\Delta_{n+2+p, q}$ and its adjacent $\Delta_{n+3+p, 4 q-\jmath}(0 \leqq j \leqq 3)$ instead of $\Delta_{n+1,4 k}$ and $\Delta_{n+2,16 k}$, and conclude that $\Delta_{n+3+p, 4 q-\jmath}$ is degenerate $(\tilde{f})$ and $\bigcup_{\ell=0}^{3} \widetilde{D}_{n+3+p, 16 q-4 \jmath-\ell} \subset\left\{w ;[w, 0] \geqq m / 2^{p+3}\right\}$. Consequently we have $(*)$ for $p+1$. Thus we see that all $\Delta_{p, q}$ contained in the end $F_{0}{ }^{\prime}$ are degenerate $(\tilde{f})$, but it is impossible as we saw in 5 . Our theorem is now established.

7. Remark. The exceptionally ramified meromorphic function $\pi \circ \phi^{-1}(z)$ considered in 3 has three totally ramified values $w_{1}=1, w_{2}=-1, w_{3}=\infty$, for which $\nu_{1}=\nu_{2}=5$ and $\nu_{3}=2$. Hence

$$
\sum_{k=1}^{3}\left(1-\frac{1}{\nu_{k}}\right)=\frac{21}{10}
$$

We compose $\{(\omega+1) /(\omega-1)\}^{1 / 5}$ with $\pi \circ \phi^{-1}(z)$. Then the composite function has five totally ramified values $w_{k}=e^{2 k \pi \imath / 5}, 0 \leqq k \leqq 4$, for which $\nu_{k}=2,0 \leqq k \leqq 4$. Hence

$$
\sum_{k=0}^{4}\left(1-\frac{1}{\nu_{k}}\right)=\frac{5}{2} \text {. }
$$

\section{REFERENCES}

[1] Ahlfors, L.V., Lectures on quasiconformal mappings. Van Nostrand (1966).

[2] Carleson, L., A remark on Picard's theorem. Bull. Amer. Math. Soc. 67 (1961), 142-144.

[3] Kurokawa, T., Exceptionally ramified meromorphic functions with a nonenumerable set of essential singularities. Nagoya Math. J. 88 (1982), 133-154.

[4] Matsumoto, K., Existence of perfect Picard sets. Nagoya Math. J. 27 (1966), 213-222.

[5] Sario, L. and Noshiro, K., Value distribution theory. Van Nostrand (1966).

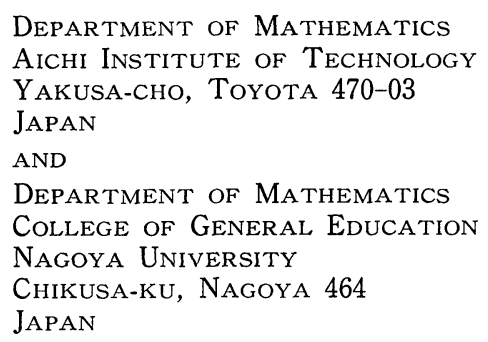

